OPEN ACCESS

Edited by: Loredana Pagano,

University of Turin, Italy

Reviewed by:

Elena Sabini,

University of Pennsylvania,

United States

Loris Bertazza,

University of Padua, Italy

*Correspondence:

YuXia

yuxiapumch@aliyun.com

Yuxin Jiang

jiangyuxinxh@163.com

${ }^{\dagger}$ These authors have contributed equally to this work

¥These authors have contributed equally to this work

Specialty section: This article was submitted to

Thyroid Endocrinology,

a section of the journal

Frontiers in Endocrinology

Received: 15 November 2021 Accepted: 22 December 2021 Published: 02 February 2022

Citation:

Gao L, Li X, Xia Y, LiU R, LiU C, Shi X, Wu Y, Ma L and Jiang $Y$ (2022) LargeVolume Lateral Lymph Node Metastasis Predicts Worse Prognosis in Papillary Thyroid Carcinoma Patients With N1b. Front. Endocrinol. 12:815207. doi: 10.3389/fendo.2021.815207

\section{Large-Volume Lateral Lymph Node Metastasis Predicts Worse Prognosis in Papillary Thyroid Carcinoma Patients With N1b}

\author{
Luying Gao ${ }^{1 \dagger}$, Xiaoyi Li ${ }^{2 \dagger}$, Yu Xia ${ }^{1 *}$, Ruifeng Liu ${ }^{1}$, Chunhao Liu ${ }^{2}$, Xinlong Shi ${ }^{1}$, \\ Yanjiao $\mathrm{Wu}^{1}{ }^{1}$, Liyuan $\mathrm{Ma}^{1}$ and Yuxin Jiang ${ }^{1 * 7}$ \\ ${ }^{1}$ Department of Ultrasound, State Key Laboratory of Complex Severe and Rare Diseases, Peking Union Medical College \\ Hospital, Chinese Academy of Medical Sciences and Peking Union Medical College, Beijing, China, ${ }^{2}$ Department of General \\ Surgery, State Key Laboratory of Complex Severe and Rare Diseases, Peking Union Medical College Hospital, Chinese \\ Academy of Medical Sciences and Peking Union Medical College, Beijing, China
}

We aimed to evaluate the relevance of large-volume lateral lymph node metastases (LLNMs) at risk of recurrence/persistence in papillary thyroid carcinoma (PTC) with LLNMs (N1b). This retrospective study included 448 PTC patients, who had positive LLNMs confirmed by histology and suspected of LLNMs by preoperative ultrasound. According to the number of pathological LLNMs, patients were divided into large-volume LLNM (number of LLNMs $>5$ ) and low-volume LLNM (number of LLNMs $\leq 5$ ). Risk factors of recurrence/persistence in PTC patients with N1b were analyzed. Preoperative ultrasound features of PTC with large-volume LLNM were analyzed. For the patients with a mean follow-up of 44.0 months, the risk of recurrence/persistence was $25.1 \%$ in PTC patients with N1b. The recurrence/persistence rate was significantly higher in patients with largevolume LLNM than in patients with low-volume LLNM by multivariate analysis (37.3\% vs. 17.1\%; HR $=2.451,95 \% \mathrm{Cl} 1.41-4.261, \mathrm{p}=0.001)$. The 3-year and 5-year recurrence/ persistence-free survival for those with large-volume LLNM were 93.2\% and 47.2\%, respectively. Moreover, we found that multilevel suspected LLNMs and loss of fatty hilum were independent preoperative indicative factors of large-volume LLNM (OR $=6.239$, 95\% Cl 3.547-10.977, $\mathrm{p}<0.001 ; \mathrm{OR}=5.149,95 \% \mathrm{Cl} 1.859-14.261, \mathrm{p}=0.002)$. In conclusion, multilevel suspected LLNM and loss of fatty hilum on ultrasound tended to be more common in patients with large-volume LLNM. PTC patients with large-volume LLNM are at a higher recurrent/persistent risk than those with low-volume LLNM. Large-volume LLNM may be used to stratify the risk of recurrence/persistence in PTC.

Keywords: papillary thyroid carcinoma, ultrasound, thyroid carcinoma, thyroid nodule, lymph node metastasis, lateral lymph node metastasis 


\section{INTRODUCTION}

The incidence of papillary thyroid carcinoma (PTC) which accounts for $80 \%-85 \%$ of thyroid cancer in iodine sufficient areas has increased worldwide (1). The appropriate use of routine surgery can reduce disease recurrence and mortality, although they might lead to treatment-related complications (2). Therefore, it is crucial to balance the potential benefit versus possible adverse effects, to accurately choose the appropriate extent of surgery and other treatments. To investigate the risk stratification and help provide the appropriate treatment for a specific patient has become the cornerstone of individualized management of PTC by our and other teams' results $(1,3,4)$. Some PTCs are associated with poor clinical characteristics, such as significant cervical lymph node metastasis (LNM) or distant metastases $(5,6)$. Lateral neck metastasis is known as an independent risk factor of loco-regional recurrence, especially in patients older than 45 years $(7-10)$. Moreover, large-volume lymph node metastasis (number of LNMs $>5$ ) has been shown to be a significant prognostic factor in the 2015 ATA guideline (1, 11-13). Central and lateral LNMs are considered to be two forms of local regional metastasis in PTC, since central lymph node metastasis (CLNM) has a lesser impact on survival (11). However, fewer studies have focused on large-volume lateral LNMs, and the utility of the number of lateral lymph node metastasis (LLNM) in the prognosis and nodal staging of PTC patients has not been comprehensively studied. Whether a significant number of LLNMs are more likely to remain or recurrence after surgery has not been confirmed.

Recent advances in ultrasound allow an accurate recognition of involved cervical compartments. In a recent meta-analysis, the sensitivity and specificity of ultrasound in detecting LLNM of PTC were $70 \%$ and $84 \%$, respectively (14). Compartmentoriented lymph node (LN) dissection instead of the extensive radical LN dissection necessitates the close cooperation of a careful examination from radiologists. However, to our knowledge, no research had studied ultrasonographic features of cervical lymph nodes for large-volume LLNM. In this study, we assessed the prognosis and ultrasound features of cervical lymph nodes for large-volume LLNM in patients with PTC.

\section{MATERIAL AND METHODS}

\section{Patients and Definitions}

We retrospectively studied all 4,306 patients who underwent thyroidectomy at our hospital between December 2012 and April 2016. The following inclusion criteria were applied: (1) patients older than 18 years of age; (2) patients with PTC confirmed via surgical pathology; (3) patients who underwent thyroidectomy with central and lateral neck dissection; (4) patients with $>5$ lateral lymph nodes removed and lateral lymph node metastases confirmed via surgical pathology; and (5) patients with preoperative ultrasound suspected of having lateral lymph node metastasis. A total of 448 patients were included (Supplementary Figure 1). During follow-up, thyroglobulin
(Tg), thyroid-stimulating hormone (TSH), and anti-Tg antibody levels were periodically measured in plasma, and ultrasound was periodically performed. If there was a suspicion of tumor recurrence/persistence, patients underwent whole-body 131iodine scan, computed tomography (CT), magnetic resonance imaging (MRI), or 18-fluorodeoxyglucose positron emission tomography (PET). In the study, recurrence/persistence was defined as persistent or newly identified locoregional or distant metastases, which were reviewed by an experienced surgeon and an experienced radiologist in combination with $\mathrm{Tg}$ level (suppressed $\mathrm{Tg}>1 \mathrm{ng} / \mathrm{ml}$ or stimulated $\mathrm{Tg}>10 \mathrm{ng} / \mathrm{ml}$ ) and $\mathrm{Tg}$ antibody status (rising $\mathrm{Tg}$ antibodies) $(1,15)$. Discrepancies were resolved through discussion.

\section{Data Collection}

All ultrasound examinations were performed using a Philips iU 22 device (Philips Healthcare, Eindhoven, Netherlands), equipped with a $5-12-\mathrm{MHz}$ linear-array transducer. The size (short axis and long axis), multifocality, shape, cystic appearance, fatty hilum, hyperechogenicity, calcifications, and vascularity of lymph nodes, were evaluated by ultrasound. The size, multifocality, and extrathyroidal extension of primary thyroid nodules were evaluated by ultrasound. Suspicious sonographic characteristics of cervical lymph nodes included loss of the fatty hilum, a rounded rather than oval shape, cystic change, hyperechogenicity, calcifications, and peripheral vascularity. In multifocal cases, the largest lymph node was analyzed. Lymph nodes were considered suspicious when one or more of the suspicious ultrasound findings were present (1). The ultrasound examinations were performed by radiologists, and the images of ultrasound were reviewed by an experienced radiologist who was blind to the patients' clinical data.

The pathological data included tumor size, extrathyroidal extension, and the status of central and lateral LNs. In multifocal cases, the largest one was analyzed. Lateral cervical LNs included levels II, III, IV, and V lymph nodes. Central cervical lymph nodes included level VI and VII lymph nodes. The number of LNMs was calculated and analyzed with respect to the neck level. Large-volume LLNM referred to more than five metastatic lateral LNs. Low-volume LLNM referred to less than or equal to five metastatic lateral LNs. Large-volume CLNM referred to more than five metastatic central LNs.

\section{Statistical analysis}

Categorical variables were presented as frequencies and analyzed using the chi-squared test. Quantitative data were presented as the mean \pm standard deviations (SDs). For parametric data, an unpaired t-test was used to evaluate the differences between the two groups. The study analyzed the median and mean recurrence/persistence-free survival using the Kaplan-Meier method and log-rank testing. Univariate Cox regression was used to evaluate the association between each of the risk factors and the study's recurrence/persistence. For multivariate analysis, the Cox proportional hazards model was used to assess the relationship between recurrence/persistence and variables. Based on the parameters from the statistically significant results of the $\chi 2$-tests, a multivariate logistic regression model was established 
to assess the correlations between sonographic features and large-volume LNM. All statistical analyses were performed with SPSS software version 19.0 (IBM, Armonk, NY, USA). Differences with $\mathrm{p}<0.05$ were considered statistically significant.

\section{RESULTS}

\section{Analysis of Risk Factors and Prognosis of PTC With Lateral Lymph Node Metastasis}

The correlation of risk factors with recurrence/persistence is shown in Table 1. The mean age of the patients at diagnosis with recurrence/persistence was $38.3 \pm 12.1$ years, and that of those without was $39.8 \pm 11.2$ years. Sex and age were not associated with recurrence/persistence ( $\mathrm{p}=0.057 ; \mathrm{p}=0.524)$. Large-volume CLNM correlated with recurrence/persistence $(\mathrm{p}=$ 0.01). $33.7 \%$ of patients with large-volume CLNM developed recurrence/persistence, compared with $18.8 \%$ of patients with low-volume CLNM. The recurrence/persistence rate was significantly higher in patients with large-volume LLNM than in patients with low-volume LLNM $(37.3 \%$ vs. $17.1 \%, \mathrm{HR}=1.85$, 95\% CI 1.23-2.77, $\mathrm{p}=0.003$ ) (Figures 1, 2). Other risk factors, such as multifocality, tumor size, extrathyroidal extension (ETE), and pathological CLNM, did not correlate with the prognosis of PTC.

To identify independent factors associated with recurrence/ persistence, variables were entered into multivariate Cox regression testing. Patients with large-volume LLNM were more likely to develop recurrence/persistence than those with low-volume LLNM $(\mathrm{HR}=2.451,95 \%$ CI 1.41-4.261, $\mathrm{p}=$ 0.001) (Table 2).

The median and mean recurrence/persistence-free survival were 60.0 and 60.6 months for the PTC patients with largevolume LLNM, respectively. The median and mean recurrence/ persistence-free survival were 69.0 and 65.5 months for the PTC patients with low-volume LLNM. The prognoses of PTC patients with large-volume LLNM were significantly worse than those with low-volume LLNM ( $\mathrm{p}=0.002)$ (Table 3).

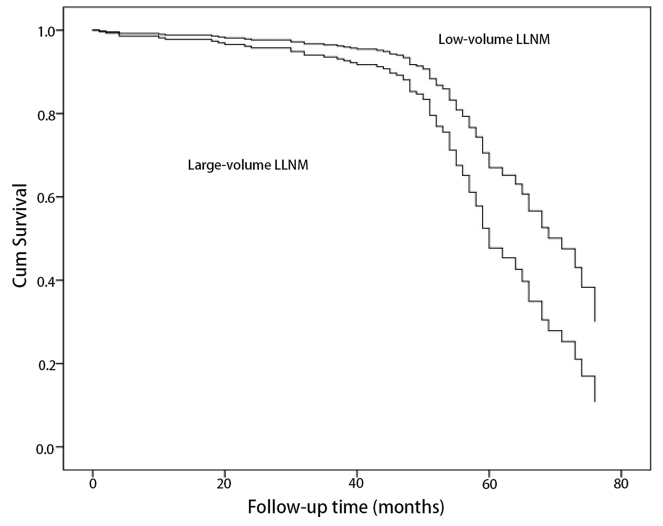

FIGURE 1 | Comparison of cumulative survival of papillary thyroid cancer patients with metastasis to large-volume lateral lymph node and low-volume lateral lymph node by univariate Cox regression $(p=0.003)$.

\section{Clinical Courses of PTC Patients With Lateral Lymph Node Metastasis}

Of the 448 patients, 387 patients $(86.4 \%)$ were followed up. The mean follow-up periods were $44.0 \pm 19.1$ months. The 3 - and 5year recurrence/persistence-free survival was $95.3 \%$ and $57.9 \%$, respectively. Recurrence/persistence was recorded in 97 patients (25.1\%), including 90 patients $(23.3 \%)$ in the regional lymph node, five patients $(1.3 \%)$ in the thyroid operative bed, and one patient $(0.3 \%)$ in the cervical soft tissue, and one patient $(0.3 \%)$ developed distant metastasis in the lung.

In our series, patients with recurrence/persistence had significantly higher abnormal $\mathrm{Tg}$ values (defined as suppressed $\mathrm{Tg}>1 \mathrm{ng} / \mathrm{ml}$ or stimulated $\mathrm{Tg}>10 \mathrm{ng} / \mathrm{ml}$ or rising $\mathrm{Tg}$ antibodies) than those without $(31 / 95,32.6 \%$ vs. $25 / 274,9.1 \%$, p < 0.001$)$ The mean serum $\mathrm{Tg}$ level in the patients with recurrence/ persistence was greater than those without $(3.34 \pm 12.05$ vs. $0.97 \pm 3.65 \mathrm{ng} / \mathrm{ml}, \mathrm{p}=0.01)$.

The mean serum Tg levels of patients with large-volume LLNM and with low-volume LLNM were $3.10 \pm 11.62$ and $0.86 \pm$

TABLE 1 | Risk factors of recurrence/persistence in PTC patients with lateral lymph node metastasis.

\begin{tabular}{|c|c|c|c|c|}
\hline & \multicolumn{2}{|c|}{ Recurrence/persistence } & \multirow[b]{2}{*}{$\mathrm{HR}(95 \% \mathrm{Cl})$} & \multirow[b]{2}{*}{$p$-value } \\
\hline & Yes & No & & \\
\hline n (\%) & $97(25.1)$ & $290(74.9)$ & & \\
\hline Male (\%) & $42(30.2)$ & $97(69.8)$ & $1.48(0.99-2.22)$ & 0.057 \\
\hline Age at diagnosis, mean $\pm S D, y r$ & $38.8 \pm 12.1$ & $40.2 \pm 11.5$ & $0.99(0.98-1.01)$ & 0.524 \\
\hline$<40$ yr $(\%)$ & $59(32.2)$ & $124(67)$ & $1.52(1.00-2.31)$ & 0.050 \\
\hline Multifocality (\%) & $77(27.8)$ & $200(72.2)$ & $1.34(0.82-2.20)$ & 0.240 \\
\hline Tumor size > $1 \mathrm{~cm}(\%)$ & $61(26.4)$ & $170(73.6)$ & $1.42(0.94-2.15)$ & 0.096 \\
\hline Pathological ETE (\%) & $60(24.5)$ & $185(75.5)$ & $1.05(0.56-1.96)$ & 0.880 \\
\hline Pathological CLNM (\%) & $94(26.6)$ & $259(73.4)$ & $3.15(0.995-9.95)$ & 0.051 \\
\hline Large-volume CLNM (\%) & $55(33.7)$ & $108(66.3)$ & $1.69(1.13-2.54)$ & 0.011 \\
\hline Large-volume LLNM (\%) & $57(37.3)$ & $96(62.7)$ & $1.85(1.23-2.77)$ & 0.003 \\
\hline Low-volume LLNM (\%) & $40(17.1)$ & $194(82.9)$ & & \\
\hline
\end{tabular}

LLNM, lateral lymph node metastasis; CLNM, central lymph node metastasis; ETE, extrathyroidal extension; yr, year; PTC, papillary thyroid cancer; Cl, confidence interval; SD, standard deviation. 


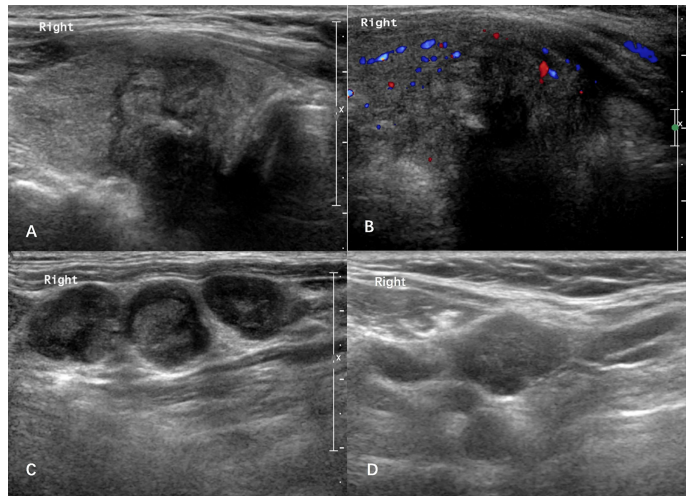

FIGURE 2 | Case: a 61-year-old man was admitted due to hoarseness. Ultrasound showed there was a $2.6-\mathrm{cm}$ solid thyroid nodule in the right lode (A, B). The right-level III lateral lymph nodes showed rounded nodes with loss of the fatty hilum (C). Histological pathology confirmed that the nodule was a papillary thyroid carcinoma with large-volume lateral lymph node metastasis in the right-level II/III/IVN neck. After a follow-up period of 59 months, the patient developed the suspected cervical lymph node recurrence in the right neck with a thyroglobulin (Tg) level of $1.8 \mathrm{ng} / \mathrm{ml}$ (D).

$3.45 \mathrm{ng} / \mathrm{ml}$, respectively. Patients with large-volume LLNM had a higher Tg level than those with low-volume LLNM ( $\mathrm{p}=0.008)$. The mean serum Tg antibody levels of patients with largevolume LLNM and with low-volume LLNM were $94.60 \pm$ 424.76 and $33.35 \pm 70.87 \mathrm{IU} / \mathrm{ml}$, respectively $(\mathrm{p}=0.11)$.

\section{Clinical Courses of PTC Patients With Large-Volume Lateral Lymph Node Metastasis}

The prevalence of large-volume LLNM was $4.1 \%$ in PTC patients and $39.5 \%$ in PTC patients with lateral lymph node metastasis (LLNM). For the PTC patients with large-volume LLNM, recurrence/persistence was found in 57 cases $(37.3 \%)$, including 53 patients $(34.6 \%)$ in the cervical lymph node, 3 patients $(2.0 \%)$ in the thyroid operative bed, and one patient $(0.7 \%)$ in cervical soft tissue. The 3- and 5-year recurrence/ persistence-free survivals were $93.2 \%$ and $47.2 \%$, respectively.

Twenty patients $(38.6 \%)$ with large-volume LLNM had recurrence/persistence lesions in the primary site of LLNM. $62.5 \%$ of patients with low-volume LLNM had recurrence/ persistence lesions in the primary site of LLNM. More patients with low-volume LLNM had recurrence/persistence lesions in the primary site of LLNM than those with large-volume LLNM $(62.5 \%$ vs. $38.6 \%, \mathrm{p}=0.003)$.

\section{Ultrasonographic Features for Large- Volume Lateral Lymph Node Metastasis}

Patients with larger tumors $(>2 \mathrm{~cm}$ ) had a significantly higher large-volume LLNM rate than those with smaller tumors $(55.7 \%$ vs. $36.5 \%, p=0.003)$. The patients with large-volume LLNM tended to involve more levels of LLNM on the US at the lateral neck compared to those with low-volume LLNM $(76.2 \%$ vs. $33.6 \%, \mathrm{p}<0.001)$. Patients with large-volume LLNM had larger
LN on the US than those with low-volume LLNM (short axis of $\mathrm{LN}, 0.93 \pm 0.55$ vs. $0.65 \pm 0.33 \mathrm{~cm}, \mathrm{p}<0.001$; long axis of LN, $1.98 \pm$ 0.96 vs. $1.46 \pm 0.71 \mathrm{~cm}, \mathrm{p}<0.001)$. Compared to low-volume metastatic LNs, large-volume metastatic LNs were more likely to have the following ultrasound features: multifocality suspected LLNM ( $46.1 \%$ vs. $21.2 \%$, p < 0.001$)$, loss of fatty hilum $(42.8 \%$ vs. $28.2 \%, \mathrm{p}<0.001)$, cystic change $(53.8 \%$ vs. $37.6 \%, \mathrm{p}=0.03)$, and peripheral vascularity $(46.1 \%$ vs. $29.6 \%, \mathrm{p}<0.001)$. There was no significant difference between large-volume and low-volume lateral LNs in terms of the ultrasound characteristics of hyperechogenicity, calcifications, and LN L/S ratio ( $p>0.05)$. Moreover, suspected CLNM on ultrasound (US) was correlated with large-volume LLNM (46.4\% vs. $34.3 \%, \mathrm{p}=0.011$ ) (Table 4).

The results of the multivariate logistic regression analysis of the suggestive factors are shown in Table 5. Multilevel suspected LLNMs and loss of fatty hilum were found to be independent factors indicative of large-volume LLNM $(\mathrm{OR}=6.239,95 \% \mathrm{CI}$ $3.547-10.977, \mathrm{p}<0.001 ; \mathrm{OR}=5.149,95 \%$ CI $1.859-14.261$, $\mathrm{p}=0.002)$

\section{DISCUSSION}

The reported overall recurrence/persistence rate of PTC ranged from $1.4 \%$ to $29.0 \%$ (7). Patients with lateral LNM are prone to have a higher recurrence/persistence rate. A previous study showed that patients with $\mathrm{N} 2$ stage $(\mathrm{LN}>3 \mathrm{~cm}$ ) were 6.18 times more likely to show lymph node recurrence than were $\mathrm{N}$-negative patients. In our study, the risk of recurrence/persistence was $25.1 \%$ in PTC patients with LLNM (pN1b). The rate was comparable to a subsequent series by Ito et al. (16), in which the risk of recurrence was $25 \%$ in clinical N1b patients with PTC $>1 \mathrm{~cm}$. A study also showed that the risk of recurrence increased to $30 \%$ in the patients that had clinical N1b disease (17). Compared with CLNM (N1a), the American Joint Committee on Cancer (AJCC) system also increases the risk of LLNM (N1b) disease, which may be due to that N1b is an indicator of more aggressive PTCs.

Some previous studies have demonstrated that the number of lymph node metastasis influenced the prognosis of thyroid cancer $(11,18-20)$. In a recent study of the SEER database, the metastasis lymph node number was significantly associated with both cancer-specific survival and overall survival in patients with differentiated thyroid cancer (11). Although the AJCC system did not use the number of LNMs to increase the risk of recurrence, five pathologically involved LNs have been shown to be a significant prognostic factor in the ATA risk stratification of recurrence (1). Compared with LLNM, CLNM has a lesser impact on survival. However, the utility of the lateral lymph node metastasis in the sub-nodal staging of PTC patients and stratifying of the patients by survival has not been shown. According to our results, recurrence/persistence was found in $37.3 \%$ of patients with large-volume LLNM. The recurrence/ persistence rate was significantly higher in patients with largevolume LLNM than in patients with low-volume LLNM by univariate and multivariate analyses. The 3- and 5-year recurrence/persistence-free survivals for those with large- 
TABLE 2 | Multivariate analysis for risk factors of recurrent/persistent status in PTC with lateral lymph node metastasis.

\begin{tabular}{|c|c|c|c|c|c|c|}
\hline & $\beta$ & SE & Wald & $\mathbf{p}$ & HR & $95 \% \mathrm{Cl}$ \\
\hline Male & 0.31 & 0.291 & 1.133 & 0.287 & 1.364 & $0.77-2.414$ \\
\hline Age $<40$ yr & 0.442 & 0.28 & 2.499 & 0.114 & 1.556 & $0.899-2.691$ \\
\hline Multifocality & 0.407 & 0.319 & 1.631 & 0.202 & 1.502 & $0.804-2.805$ \\
\hline Tumor size $>1 \mathrm{~cm}$ & -0.045 & 0.288 & 0.024 & 0.877 & 0.956 & $0.544-1.682$ \\
\hline Pathological ETE & 0.387 & 0.278 & 1.932 & 0.164 & 1.472 & $0.853-2.539$ \\
\hline Large-volume CLNM & 0.5 & 0.284 & 3.097 & 0.078 & 1.648 & $0.945-2.876$ \\
\hline Large-volume LLNM & 0.897 & 0.282 & 10.101 & 0.001 & 2.451 & $1.41-4.261$ \\
\hline
\end{tabular}

LLNM, lateral lymph node metastasis; CLNM, central lymph node metastasis; Cl, confidence interval; OR, odds ratio; PTC, papillary thyroid cancer; ETE, extrathyroidal extension; yr, year; $S D$, standard deviation.

TABLE 3 | The median and mean recurrence/persistence -free survival time of the PTC patients with large-volume lateral lymph node metastasis and low-volume lateral lymph node metastasis.

Median survival time, months $(95 \% \mathrm{Cl})$

Mean survival time, months $(95 \% \mathrm{Cl})$

\begin{tabular}{ll}
\hline Large-volume LLNM & $60(56.67-63.33)$ \\
Low-volume LLNM & $69(63.01-74.99)$
\end{tabular}

PTC, papillary thyroid cancer; LLNM, lateral lymph node metastasis.

TABLE 4 | Ultrasonographic features for large-volume lateral lymph node metastasis.

\begin{tabular}{|c|c|c|c|}
\hline & Low-volume LLNM & Large-volume LLNM & p-value \\
\hline n (\%) & $271(60.5)$ & $177(39.5)$ & \\
\hline Tumor size, mean $\pm \mathrm{SD}, \mathrm{cm}$ & $1.28 \pm 0.72$ & $1.58 \pm 0.91$ & $<0.001$ \\
\hline$>2 \mathrm{~cm}(\%)$ & $31(44.3)$ & $39(55.7)$ & 0.003 \\
\hline ETE on US (\%) & $19(51.4)$ & $18(48.6)$ & 0.248 \\
\hline Multifocality suspected LLNMs on US (\%) & $178(53.9)$ & $152(46.1)$ & $<0.001$ \\
\hline Multilevel suspected LLNMs & $91(40.3)$ & $135(59.7)$ & $<0.001$ \\
\hline Loss of the fatty hilum (\%) & $210(57.2)$ & $157(42.8)$ & $<0.001$ \\
\hline Hyperechogenicity (\%) & $11(42.3)$ & $15(57.7)$ & 0.06 \\
\hline Cystic change (\%) & $24(46.2)$ & $28(53.8)$ & 0.03 \\
\hline Calcifications (\%) & $113(55.9)$ & $89(44.1)$ & 0.09 \\
\hline Peripheral vascularity (\%) & $145(53.9)$ & $124(46.1)$ & $<0.001$ \\
\hline LLN short axis, mean $\pm \mathrm{SD}, \mathrm{cm}$ & $0.65 \pm 0.33$ & $0.93 \pm 0.55$ & $<0.001$ \\
\hline LLN long axis, mean $\pm \mathrm{SD}, \mathrm{cm}$ & $1.46 \pm 0.71$ & $1.98 \pm 0.96$ & $<0.001$ \\
\hline LLN L/S & $2.46 \pm 1.04$ & $2.35 \pm 0.93$ & 0.26 \\
\hline$<2(\%)$ & $91(59.9)$ & $61(40.1)$ & 0.65 \\
\hline Suspected CLNM on US (\%) & $103(53.6)$ & $89(46.4)$ & 0.011 \\
\hline
\end{tabular}

LLNM, lateral lymph node metastasis; CLNM, central lymph node metastasis; LLN, lateral lymph node; US, ultrasound; LLN, lymph node; L/S, long/short; ETE, extrathyroidal extension; SD: standard deviation.

TABLE 5 | Multivariate analysis of the sonographic features of large-volume lateral lymph node metastasis.

\begin{tabular}{|c|c|c|c|c|c|c|}
\hline & $\beta$ & SE & Wald & $\mathbf{p}$ & OR & $95 \% \mathrm{Cl}$ \\
\hline Tumor size $>2 \mathrm{~cm}$ & 0.658 & 0.337 & 3.823 & 0.051 & 1.932 & 0.998-3.737 \\
\hline ETE & -0.092 & 0.741 & 0.015 & 0.901 & 0.912 & $0.213-3.901$ \\
\hline Multifocality suspected LLNMs & 0.009 & 0.374 & 0.001 & 0.982 & 1.009 & $0.485-2.1$ \\
\hline Multilevel suspected LLNMs & 1.831 & 0.288 & 40.352 & $<0.001$ & 6.239 & $3.547-10.977$ \\
\hline Loss of the fatty hilum of $L N$ & 1.639 & 0.52 & 9.943 & 0.002 & 5.149 & $1.859-14.261$ \\
\hline Cystic change of LN & 0.583 & 0.384 & 2.303 & 0.129 & 1.792 & $0.844-3.807$ \\
\hline Calcifications of LN & 0.401 & 0.263 & 2.33 & 0.127 & 1.493 & $0.892-2.499$ \\
\hline Peripheral vascularity of $L N$ & 0.361 & 0.289 & 1.563 & 0.211 & 1.434 & $0.815-2.525$ \\
\hline LLN L/S $<2$ & -0.311 & 0.265 & 1.378 & 0.24 & 0.733 & $0.436-1.232$ \\
\hline Suspected CLNM & 0.426 & 0.26 & 2.688 & 0.101 & 1.532 & $0.92-2.55$ \\
\hline Constant & -3.628 & 0.606 & 35.847 & 0 & 0.027 & \\
\hline
\end{tabular}

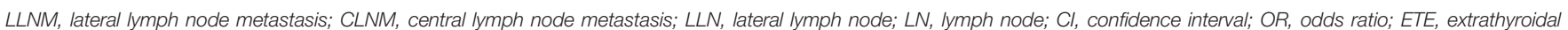
extension; L/S, long/short. 
volume LLNM were $93.2 \%$ and $47.2 \%$, respectively. Similarly, a previous study of patients with clinical N1b disease also showed that having 5 or more clinically apparent metastases independently affected disease-free survival, which may have a limited sensitivity since more than half of pathologically confirmed LNMs can be missed by preoperative clinical evaluation (18). Our results presented additional information indicating that the presence of large-volume LNM in the lateral neck $(\mathrm{N} 1 \mathrm{~b})$ is important data for postoperative recurrence risk stratification. The findings may be useful for thyroid cancer recurrence classification modifying.

Several studies defined the disease recurrence of thyroid cancer as evidence of disease following a remission period of 1 or 2 years (7). The concept of PTC persistence or recurrence after the operation is still difficult to distinguish because of its indolent nature (15). The persistent or recurrent lesions in the study refer to new lesions or residual lesions found during the follow-up after surgery. $38.6 \%$ of patients with large-volume LLNM had recurrence/persistence lesions in the primary location of LLNM, and most of the patients (84.2\%) had recurrence/persistence lesions in the lateral neck. Moreover, we found that more patients with lowvolume LLNM had recurrence/persistence lesions in the primary site of LLNM than those with large-volume LLNM (62.5\% vs. $38.6 \%$ ). We speculate that it may be due to the patients with largevolume LLNM being more aggressive and prone to metastasis, or patients with low-volume LLNM having persistent lymph node lesions after the initial surgery.

Due to the deep location of central compartment LNs, it is difficult to identify features of these LNs on ultrasound. Preoperative neck ultrasound has a low sensitivity $(48.1 \%)$ for detecting CLN metastasis. Ultrasound is the preferred screening technique in the preoperative assessment of lateral neck lymph nodes with a sensitivity of $70 \%-80 \%(14,21)$. Accurate and reliable preoperative ultrasound knowledge of these lateral metastatic lymph nodes will allow an individualized surgical approach (22). We explored the differences in preoperative ultrasound features for predicting large-volume LLNM. In our investigation, multilevel suspected LLNMs and loss of fatty hilum of LN were independently associated with large-volume LLNM. Although the loss of fatty hilum on ultrasound was associated with large-volume LLNM, the overall interobserver agreement of loss of fatty hilum was not satisfied $(\kappa=0.51)(23)$. The PTC patients with multilevel suspected LLNMs may be considered to perform more aggressive subsequent treatment for the lateral lymph node.

Our study has several limitations, including the retrospective study design and the mean follow-up time of 44 months, which was not a long period. Moreover, the size of the pathological lateral lymph nodes was not assessed by postoperative pathology. Thirdly, as ultrasound examination is real-time and operatordependent, and the ultrasound characteristic evaluation in the study may be influenced by the operators. Finally, in the study recurrence/persistence was defined as persistent or newly identified locoregional or distant metastases in combination with the Tg level and $\mathrm{Tg}$ antibody status, which was not confirmed through cytology. Future studies are needed to confirm and extend our results.

\section{CONCLUSION}

PTC patients with large-volume lateral lymph node metastasis were at a significantly higher recurrence/persistence risk than those with low-volume lateral lymph node metastasis (at least in the short-term follow-up). Large-volume lateral lymph node metastasis may be considered in the nodal staging for the prognosis risk stratification of PTC patients. Since the patients with large-volume LLNM tended to involve multilevel suspected LLNMs and LNs with loss of fatty hilum, these preoperative ultrasound findings may be useful for identifying patients at higher large-volume LLNM risk.

\section{DATA AVAILABILITY STATEMENT}

The raw data supporting the conclusions of this article will be made available by the authors, without undue reservation.

\section{ETHICS STATEMENT}

The studies involving human participants were reviewed and approved by the ethics committee of Peking Union Medical College Hospital. Written informed consent for participation was not required for this study in accordance with the national legislation and the institutional requirements. Written informed consent was obtained from the individual(s) for the publication of any potentially identifiable images or data included in this article.

\section{AUTHOR CONTRIBUTIONS}

YX and XL conceived of and designed the study. LG was the major contributor in performing the analysis, writing the manuscript, and preparing the figures and tables. YJ, CL, and XL participated in the study design and edited the manuscript. RL, XS, LM, and YW participated in the verification of data, image quality verification, selection, and collection of samples. All authors contributed to the article and approved the submitted version.

\section{FUNDING}

This study was funded by the Tibet Autonomous Region Science and Technology Project (XZ201901-GB-04), Tibet Autonomous Region Organization and Aiding Project (XZ2019ZR ZY05(Z)), and Peking Union Medical College Hospital (0104170).

\section{SUPPLEMENTARY MATERIAL}

The Supplementary Material for this article can be found online at: https:/www.frontiersin.org/articles/10.3389/fendo.2021.815207/ full\#supplementary-material

Supplementary Figure 1 | Flowchart of patient selection and inclusion in the study. 


\section{REFERENCES}

1. Haugen BR, Alexander EK, Bible KC, Doherty GM, Mandel SJ, Nikiforov YE, et al. American Thyroid Association Management Guidelines for Adult Patients With Thyroid Nodules and Differentiated Thyroid Cancer: The American Thyroid Association Guidelines Task Force on Thyroid Nodules and Differentiated Thyroid Cancer. Thyroid (2016) 26:1-133. doi: 10.1089/ thy.2015.0020

2. Pacini F, Schlumberger M, Dralle H, Elisei R, Smit JW, Wiersinga W, et al. European Consensus for the Management of Patients With Differentiated Thyroid Carcinoma of the Follicular Epithelium. Eur J Endocrinol (2006) 154:787-803. doi: 10.1530/eje.1.02158

3. Huang XP, Ye TT, Zhang L, Liu RF, Lai XJ, Wang L, et al. Sonographic Features of Papillary Thyroid Microcarcinoma Predicting High-Volume Central Neck Lymph Node Metastasis. Surg Oncol (2018) 27:172-6. doi: 10.1016/j.suronc.2018.03.004

4. Gao L, Wang J, Jiang Y, Gao Q, Wang Y, Xi X, et al. The Number of Central Lymph Nodes on Preoperative Ultrasound Predicts Central Neck Lymph Node Metastasis in Papillary Thyroid Carcinoma: A Prospective Cohort Study. Int J Endocrinol (2020) 14:2698659. doi: 10.1155/2020/2698659

5. Siddiqui S, White MG, Antic T, Grogan RH, Angelos P, Kaplan EL, et al. Clinical and Pathologic Predictors of Lymph Node Metastasis and Recurrence in Papillary Thyroid Microcarcinoma. Thyroid (2016) 26:807-15. doi: 10.1089/thy.2015.0429

6. Lundgren CI, Hall P, Dickman PW, Zedenius J. Clinically Significant Prognostic Factors for Differentiated Thyroid Carcinoma: A Population-Based, Nested CaseControl Study. Cancer (2006) 106:524-31. doi: 10.1002/cncr.21653

7. Suh YJ, Kwon H, Kim SJ, Choi JY, Lee KE, Park YJ, et al. Factors Affecting the Locoregional Recurrence of Conventional Papillary Thyroid Carcinoma After Surgery: A Retrospective Analysis of 3381 Patients. Ann Surg Oncol (2015) 22:3543-9. doi: 10.1245/s10434-015-4448-9

8. Ito Y, Miyauchi A, Kihara M, Kobayashi K, Miya A. Prognostic Values of Clinical Lymph Node Metastasis and Macroscopic Extrathyroid Extension in Papillary Thyroid Carcinoma. Endocr J (2014) 61:745-50. doi: 10.1507/ endocri.EJ14-0138

9. Verburg FA, Mäder U, Tanase K, Thies ED, Diessl S, Buck AK, et al. Life Expectancy is Reduced in Differentiated Thyroid Cancer Patients $\geq 45$ Years Old With Extensive Local Tumor Invasion, Lateral Lymph Node, or Distant Metastases at Diagnosis and Normal in All Other DTC Patients. J Clin Endocrinol Metab (2013) 98:172-80. doi: 10.1210/jc.2012-2458

10. Machens A, Dralle H. Correlation Between the Number of Lymph Node Metastases and Lung Metastasis in Papillary Thyroid Cancer. J Clin Endocrinol Metab (2012) 97:4375-82. doi: 10.1210/jc.2012-1257

11. Wei WJ, Lu ZW, Wen D, Liao T, Li DS, Wang Y, et al. The Positive Lymph Node Number and Postoperative N-Staging Used to Estimate Survival in Patients With Differentiated Thyroid Cancer: Results From the Surveillance, Epidemiology, and End Results Dataset (1988-2008). World J Surg (2018) 42:1762-71. doi: 10.1007/s00268-017-4343-6

12. Randolph GW, Duh QY, Heller KS, LiVolsi VA, Mandel SJ, Steward DL, et al. The Prognostic Significance of Nodal Metastases From Papillary Thyroid Carcinoma can be Stratified Based on the Size and Number of Metastatic Lymph Nodes, as Well as the Presence of Extranodal Extension. Thyroid (2012) 22:1144-52. doi: 10.1089/thy.2012.0043

13. Liu C, Liu Y, Zhang L, Dong Y, Hu S, Xia Y, et al. Risk Factors for LargeVolume Lymph Node Metastases in Cn0 Papillary Thyroid Microcarcinoma. Gland Surg (2019) 8:550-6. doi: 10.21037/gs.2019.10.04

14. Zhao H, Li H. Meta-Analysis of Ultrasound for Cervical Lymph Nodes in Papillary Thyroid Cancer: Diagnosis of Central and Lateral Compartment
Nodal Metastases. Eur J Radiol (2019) 112:14-21. doi: 10.1016/ j.ejrad.2019.01.006

15. Chinese Society of Clinical Oncology (CSCO) Diagnosis and Treatment Guidelines for Persistent/Recurrent and Metastatic Differentiated Thyroid Cancer Working Group: (2019) Chinese Society of Clinical Oncology (CSCO) Diagnosis and Treatment Guidelines for Persistent/Recurrent and Metastatic Differentiated Thyroid Cancer 2018 (English Version). Chin J Cancer Res (2019) 31:99-116. doi: 10.21147/j.issn.1000-9604.2019.01.06

16. Ito $\mathrm{Y}$, Jikuzono $\mathrm{T}$, Higashiyama $\mathrm{T}$, Asahi $\mathrm{S}$, Tomoda $\mathrm{C}$, Takamura $\mathrm{Y}$, et al. Clinical Significance of Lymph Node Metastasis of Thyroid Papillary Carcinoma Located in One Lobe. World J Surg (2006) 30:1821-8. doi: 10.1007/s00268-006-0211-5

17. Moreno MA, Agarwal G, de Luna R, Siegel ER, Sherman SI, Edeiken-Monroe BS, et al. Preoperative Lateral Neck Ultrasonography as a Long-Term Outcome Predictor in Papillary Thyroid Cancer. Arch Otolaryngol Head Neck Surg (2011) 137:157-62. doi: 10.1001/archoto.2010.254

18. Ito $\mathrm{Y}$, Fukushima $\mathrm{M}$, Tomoda $\mathrm{C}$, Inoue $\mathrm{H}$, Kihara $\mathrm{M}$, Higashiyama $\mathrm{T}$, et al. Prognosis of Patients With Papillary Thyroid Carcinoma Having Clinically Apparent Metastasis to the Lateral Compartment. Endocr J (2009) 56(6):75966. doi: 10.1507/endocrj.K09E-025

19. So YK, Kim MJ, Kim S, Son YI. Lateral Lymph Node Metastasis in Papillary Thyroid Carcinoma: A Systematic Review and Meta-Analysis for Prevalence, Risk Factors, and Location. Int J Surg (2018) 50:94-103. doi: 10.1016/j.jisu.2017.12.029

20. Sapuppo G, Palermo F, Russo M, Tavarelli M, Masucci R, Squatrito S, et al. Latero-Cervical Lymph Node Metastases (N1b) Represent an Additional Risk Factor for Papillary Thyroid Cancer Outcome. J Endocrinol Invest (2017) 40:1355-63. doi: 10.1007/s40618-017-0714-y

21. Khokhar MT, Day KM, Sangal RB, Ahmedli NN, Pisharodi LR, Beland MD, et al. Preoperative High-Resolution Ultrasound for the Assessment of Malignant Central Compartment Lymph Nodes in Papillary Thyroid Cancer. Thyroid (2015) 25:1351-4. doi: 10.1089/thy.2015.0176

22. Sakorafas GH, Koureas A, Mpampali I, Balalis D, Nasikas D, Ganztzoulas S. Patterns of Lymph Node Metastasis in Differentiated Thyroid Cancer; Clinical Implications With Particular Emphasis on the Emerging Role of Compartment-Oriented Lymph Node Dissection. Oncol Res Treat (2019) 42:143-7. doi: 10.1159/000488905

23. Ryu KH, Lee KH, Ryu J, Baek HJ, Kim SJ, Jung HK, et al. Cervical Lymph Node Imaging Reporting and Data System for Ultrasound of Cervical Lymphadenopathy: A Pilot Study. AJR Am J Roentgenol (2016) 206:128691. doi: 10.2214/AJR.15.15381

Conflict of Interest: The authors declare that the research was conducted in the absence of any commercial or financial relationships that could be construed as a potential conflict of interest.

Publisher's Note: All claims expressed in this article are solely those of the authors and do not necessarily represent those of their affiliated organizations, or those of the publisher, the editors and the reviewers. Any product that may be evaluated in this article, or claim that may be made by its manufacturer, is not guaranteed or endorsed by the publisher.

Copyright $\odot 2022 \mathrm{Gao}, \mathrm{Li}, \mathrm{Xia}, \mathrm{Liu}, \mathrm{Liu}, \mathrm{Shi}, \mathrm{Wu}, \mathrm{Ma}$ and Jiang. This is an openaccess article distributed under the terms of the Creative Commons Attribution License (CC BY). The use, distribution or reproduction in other forums is permitted, provided the original author(s) and the copyright owner(s) are credited and that the original publication in this journal is cited, in accordance with accepted academic practice. No use, distribution or reproduction is permitted which does not comply with these terms. 